\title{
Molecular Bases for Combinatorial Treatment Strategies in Patients with KRAS Mutant Lung Adenocarcinoma and Squamous Cell Lung Carcinoma
}

\author{
Chiara Lazzari · Alberto Verlicchi · Anastasios Gkountakos · Sara Pilotto • \\ Mariacarmela Santarpia - Imane Chaib - Jose Luis Ramirez Serrano - Santiago Viteri · \\ Daniela Morales-Espinosa · Claudio Dazzi · Filippo de Marinis · Peng Cao • \\ Niki Karachaliou · Rafael Rosell
}

Received: January 15, 2016 / Published online: March 18, 2016

(C) The Author(s) 2016. This article is published with open access at Springerlink.com

\section{ABSTRACT}

Innovative therapeutic agents have significantly improved outcomes, with an acceptable safety profile, in a substantial proportion of non-small cell lung cancer (NSCLC) patients in whom the malignant phenotype of the disease is determined by oncogenic molecular alterations. However, the benefit seen with these treatment models has not translated well

Enhanced content To view enhanced content for this article go to http://www.medengine.com/Redeem/ CB84F06056933F05

C. Lazzari · F. de Marinis

Divisione di Oncologica Toracica, Istituto Europeo di Oncologia, Milan, Italy

A. Verlicchi · C. Dazzi

Oncology, Ospedale Sta Maria delle Croci, Ravenna, Italy

A. Gkountakos

Laboratory of Tumor Cell Biology, School of

Medicine, University of Crete, Heraklion, Greece

S. Pilotto

Medical Oncology, Azienda Ospedaliera

Universitaria Integrata, University of Verona,

Verona, Italy

M. Santarpia

Medical Oncology Unit, Human Pathology

Department, University of Messina, Messina, Italy to NSCLCs with KRAS mutations or squamous cell histology. Although efforts have been made to develop precision medicine approaches, KRAS mutant NSCLC and lung squamous cell carcinoma (LSCC) continue to display resistance to therapy. Recently, based on the results of the Phase III SQUIRE trial, the EGFR monoclonal antibody necitumumab received FDA authorization in combination with cisplatin and gemcitabine for first line treatment of patients with metastatic LSCC. Among the molecular compounds tested in KRAS mutant NSCLC patients, the MEK

I. Chaib · J. L. Ramirez Serrano - R. Rosell Institut d'Investigació en Ciències Germans Trias i Pujol, Badalona, Spain

S. Viteri - D. Morales-Espinosa - N. Karachaliou • R. Rosell

Instituto Oncológico Dr. Rosell (IOR),

Quirón-Dexeus University Institute, Barcelona, Spain

P. Cao

Laboratory of Cellular and Molecular Biology, Jiangsu Province Academy of Traditional Chinese Medicine, Nanjing, China

R. Rosell $(\bowtie) \cdot J$. L. Ramirez Serrano Cancer Biology and Precision Medicine Program, Catalan Institute of Oncology (ICO), Hospital Germans Trias i Pujol, Badalona, Spain e-mail: rrosell@iconcologia.net 
inhibitor, selumentinib, combined with docetaxel in second line setting, determined a progression-free survival improvement, but no overall survival advantage. Better understanding is needed in regard to signaling pathways which cooperate to induce oncogene transformation in LSCC and KRAS mutant NSCLC and could determine intrinsic or acquired resistance to necitumumab and selumetinib. Greater understanding of such pathways will provide a molecular base upon which to improve the scant clinical benefit with these compounds.

Keywords: KRAS; Necitumumab; Non-small cell lung cancer; Selumetinib; Squamous lung cancer

\section{INTRODUCTION}

Over the past 10 years, the discovery of molecular alterations susceptible to targeted inhibition has significantly improved overall survival (OS) in a small group of non-small cell lung cancer (NSCLC) patients [1-5]. However, subgroups of oncogenic drivers that are targetable are not common [6], and overall treatment outcomes of NSCLC patients remain unsatisfactory, with low long-term survival rates.

Direct inhibition of Kirsten Rat Sarcoma Viral oncogene homolog (KRAS), the most commonly mutated oncogene in lung adenocarcinoma, has proven clinically challenging [7]. The KRAS gene is located in the short arm of chromosome 12 and encodes for two splice variants, KRAS4A and KRAS4B [8]. Mutated KRAS has a putative role in NSCLC, most frequently observed in tumors arising in smokers with adenocarcinoma histology $[9,10]$. RAS mediates the intracellular signaling pathway in response to activation of cell surface receptors. Once activated, a tyrosine kinase receptor binds to an adaptor protein, Grb2, which recruits the guanine nucleotide exchange factor SOS. SOS facilitates the RAS GDP-GTP exchange, leading to RAS activation, which in turns stimulates the RAF-MEK-ERK mitogen-activated protein kinase (MAPK), the phosphatidylinositol 3-kinase (PI3K)-AKTmTOR and the RAS-like (RAL) pathways (Fig. 1). RAS signaling is switched off by GTPase activating proteins (GAPs) responsible for GTP hydrolysis [11]. KRAS mutations, which impair GTP hydrolysis, are located in the sites involved in GAP binding, including codons 12 , 13 and 61, with the amino acid substitution G12C being the most common [7, 9-11].

Many efforts have been made to find treatment strategies able to inhibit downstream effectors of KRAS. Recently, a phase II study of combination docetaxel plus MEK inhibitor selumetinib compared with docetaxel and placebo in 87 NSCLC patients, who had previously failed with first line therapy, showed a statistically significant progression-free survival (PFS) and objective response rate (ORR) improvement in favor of selumetinib, with no overall survival (OS) advantage [12]. The heterogeneous biology of KRAS mutant NSCLC tumors may partially explain the difficulties encountered in the development of efficient therapies. It is still a matter of debate whether and how the distinct oncogenic RAS mutations may affect the biological and clinical behavior of KRAS mutant patients. The hydrophobic G12C and G12V activate the RAL pathway, while the hydrophilic G12D acts through PI3K-AKT signaling [13]. Defining whether cells are KRAS dependent or independent for tumor growth is also of great relevance [14]. Co-occuring genetic alterations in genes other than KRAS may 


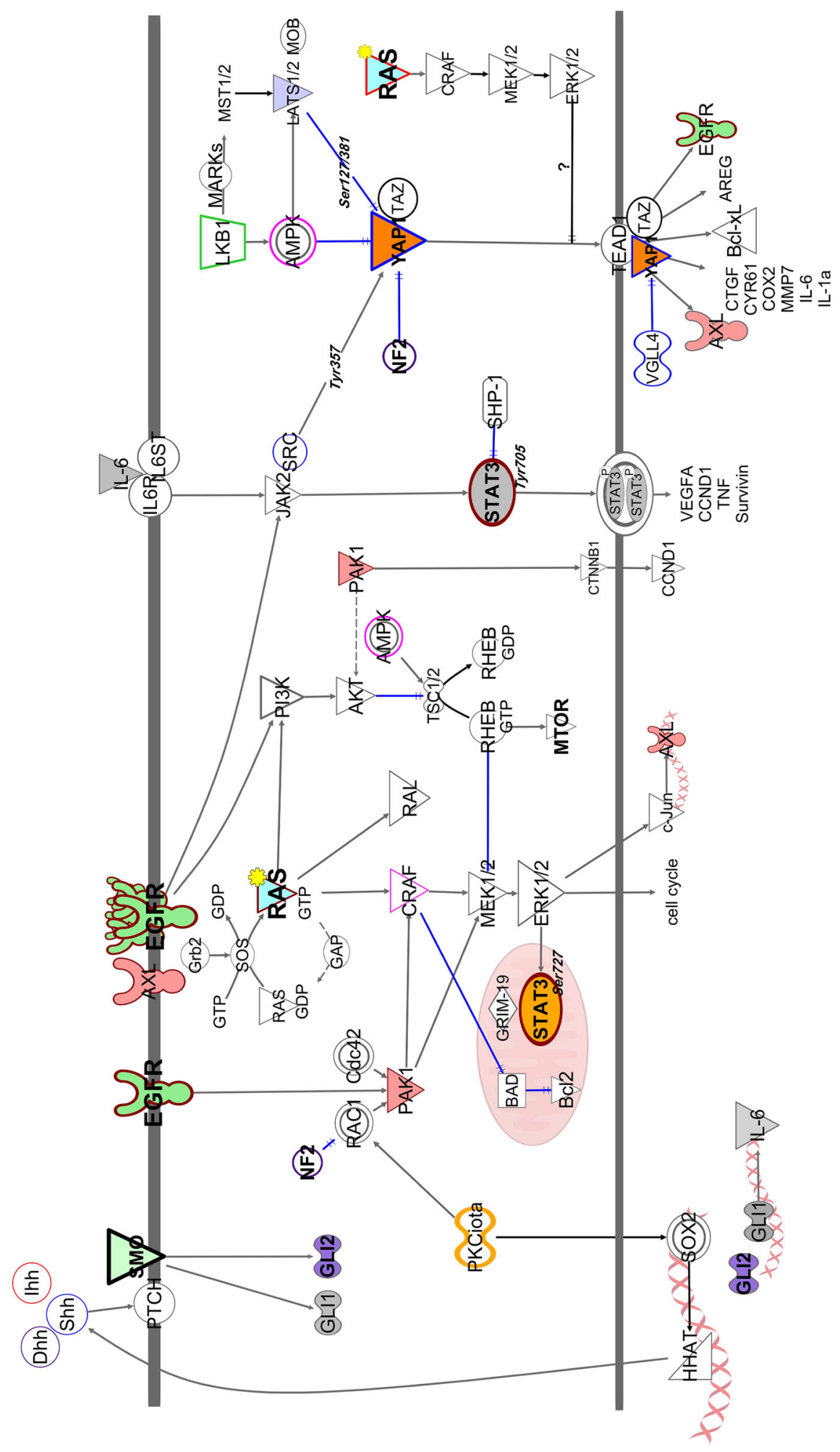


4 Fig. 1 Signal transduction pathways in KRAS mutant NSCLC and LSCC. RAS is the downstream effector of the activated tyrosine kinase receptor EGFR. SOS favors the GDP-GTP exchange, with the subsequent activation of three intracellular signaling pathways: (1) CRAF-MEK-ERK; (2) PI3K-AKT-mTOR; (3) RAL. Mutations in RAS determine its constitutive activation. ERK and AKT signaling are also stimulated by the serine/threonine kinase PAK1, which is regulated by the small GTPases RAC1 (upon PKCiota) and Cdc42. PAK1 phosphorylates CRAF at Ser338, which translocates into mitochondria and inhibits BAD, suppressing apoptosis, and MEK1 at Ser298, with the subsequent expression of cyclin D1, a key driver for cell cycle progression. PAK1 recruits AKT to plasma membrane, allowing its activation, which stimulates mTOR. mTOR is also augmented by the serine/threonine kinase LKB1, which activates AMPK, responsible for RHEB inhibition, promoting mTOR activity. LKB1 is an additional indirect regulator of the transcriptional co-activator YAP1, which induces transcription of $\mathrm{BCL}-\mathrm{xL}$, CTGF, CYR61, COX2, MMP7, IL-6, IL-1 $\alpha$ and AXL through binding to TEAD transcription factors. LKB1 phosphorylates LATS at Thr1079, which is responsible for YAP1 phosphorylation at Ser127 and Ser381, favoring its retention into the cytoplasm and its proteasomal degradation. LATS is phosphorylated by MST1 upon MARK activation. YAP1 is also activated by the Src family kinase Yes, or Jun $\mathrm{N}$ terminal kinases (JUNK), and inhibited by NF2, which phosphorylates YAP1, preventing its translocation into the nucleus. NF2 additionally inhibits RAC1, blocking CRAF and MEK phosphorylation mediated by PAK1. Phosphorylation of Tyr705 of cytoplasmic STAT3 in response to activated EGFR promotes STAT3 homodimerization, which leads to nucleus translocation and DNA binding. IL-6 by JAK2 phosphorylation favors STAT3 recruitment. ERK phosphorylates STAT3 on Ser727 which subsequently interacts with GRIM-19. This interaction favors STAT3 transportation and anchorage to the inner mitochondrial membrane. Shh indirectly promotes STAT3 activation, as it induces the binding between Gli1 and IL-6 promoter. Shh is regulated by $\mathrm{PTCH}$ and SMO. Upon Hh ligand binding to PTCH, SMO initiates a signaling cascade that culminates in the activation of GLI transcription factors. In the absence of ligands, PATCH represses SMO activity

promote cancer cell proliferation and survival and contribute to the development of escape mechanisms to MEK inhibition [15].

By contrast, progress in the field of lung squamous cell carcinoma (LSCC), representing about $30 \%$ of NSCLC cases, has lagged behind. Although molecular alterations in LSCC have been described, effective targeted therapies have not yet been developed [16]. Standard treatment for LSCC is currently based on platinum doublets in the first line, and monochemotherapy or immunotherapy in subsequent lines [17, 18]. Different potentially targetable molecular alterations have been identified in LSCC tumors, including phosphoinositide 3-kinase (PIK3CA), fibroblast growth factor receptor 1 (FGFR1), or c-MET amplification, and discoidin domain receptor tyrosine kinase 2 (DDR2) mutations, although none of these biomarkers have yet been validated in the clinical setting [19]. The epidermal growth factor receptor (EGFR) gene is commonly overexpressed in patients with LSCC [20], and two monoclonal anti-EGFR antibodies, cetuximab and necitumumab, have been tested in phase III studies. The FLEX trial demonstrated superior OS for chemotherapy plus cetuximab compared to chemotherapy alone for patients with advanced EGFR-expressing NSCLC, with the greatest survival benefit observed in the subgroup of patients with squamous histology [21]. A non-preplanned analysis, performed to build an immunohistochemistry score for EGFR expression, confirmed that high EGFR levels are more commonly detected in LSCC than in other types of NSCLC [22]. It was not possible to fully validate these results in a second phase III trial (BMS099), in which cetuximab showed no benefit in OS, progression-free survival (PFS) or response in combination with first line carboplatin and docetaxel [23]. In the SQUIRE trial, necitumumab plus cisplatin and gemcitabine was compared to cisplatin and gemcitabine alone for advanced LSCC. The study met its primary endpoint, 
showing a statistically significant benefit in OS with necitumumab. Moreover, a preplanned analysis showed that patients with EGFR overexpressing tumors benefited most from necitumumab [24]. Based on these results, in November 2015, the Food and Drug Administration (FDA) in the US approved necitumumab in combination with cisplatin and gemcitabine for first line treatment of patients with metastatic LSCC. However, acquired resistance eventually develops, while the lack of clearly defined predictive biomarkers to optimize patient selection remains the main limitation for the use of necitumumab.

Here, we describe the intracellular signaling pathways that may have an impact on the development of KRAS mutant NSCLC and LSCC tumors, and can represent druggable targets to be combined with selumetinib or necitumumab, and define synthetic lethal approaches. We focus on common signaling pathways activated in both KRAS mutant and LSCC tumors, which dictate distinct pathologic outputs and favor the occurrence of two distinct pathologic entities. Since tumor cells use common signaling pathways for growth, proliferation and migration, information regarding tumors other than lung is also included. Common alterations previously described in LSCC, such as those affecting members of the PI3K pathway, or amplification of fibroblast growth factor receptor 1 or mutations in the discoidin domain receptor 2 kinase gene are not the main objective of this review [25]. This article is based on previously conducted studies and does not involve any new studies of human or animal subjects performed by any of the authors.

\section{REVISITING STAT3 SIGNALING IN KRAS MUTANT NSCLC AND LSCC}

Deregulation of signal transducer and activator of transcription (STAT3) has been observed in different tumors [26]. Phosphorylation at tyrosine (Tyr) 705, in response to growth factors and cytokines, is required to activate STAT3. Upon Janus kinase (JAK) activation, growth factor receptors or cytokine receptors create docking sites to recruit cytoplasmic STAT3 which dimerizes, translocates into the nucleus and promotes the transcription of genes involved in cell cycle progression and apoptosis (Fig. 1) [27].

Preclinical data showed that phosphorylated STAT3 increases following MEK inhibition in KRAS mutant cells [28]. STAT3 contributes to drug resistance through activation of FGFR and JAK. Combinatorial treatment with the dual JAK1 and FGFR inhibitor ponatinib and the MEK inhibitor selumetinib suppressed STAT3 phosphorylation in vitro [28]. Similar results were observed in vivo, in which concomitant treatment with either ponatinib and GDC0973 (MEK inhibitor), or ruxolitinib (JAK1/2 inhibitor) with GDC0973, or the triple combination of ruxolitinib, ponatinib and GDC0973, significantly reduced tumor growth [28]. By exploring the pathogenic contribution of STAT3 in KRAS mutant tumors, Brooks et al. found that augmented levels of only interleukin 6 (IL-6) and its essential trans-signaling receptor subunit, sIL-6R, are observed in lung adenocarcinoma patients, and are also associated with exacerbated KRAS G12D-driven lung carcinogenesis in mice [29].

Another emerging key role of STAT3 is related to its mitochondrial activation upon 
serine (Ser) 727 phosphorylation, which is critical in inducing KRAS oncogene transformation [30]. Extracellular signal-regulated kinase (ERK) is responsible for Ser727 phosphorylation (Fig. 1) [31]. Once activated, mitochondrial STAT3 stimulates the mitochondrial electron transport chain and regulates tumor cell metabolism [32]. Gene-associated retinoid-IFN-induced mortality-19 (GRIM-19) interacts with Ser727 phosphorylated STAT3 and favors its anchorage to the inner mitochondrial membrane [33]. Mitochondrial membrane potential is essential to maintain KRAS-transformed cells. Loss of phosphorylated Ser727 STAT3 is associated with reduced activity of succinate oxidoreductase and ATP synthase [30]. Lack of STAT3 decreases ATP cellular production and colony formation is largely abolished, even in the presence of the RAF-MEK-ERK pathway [30].

STAT3 represents an appealing target in LSCC, particularly due to important interactions with the EGFR signaling pathway [34]. STAT3 inhibition by cucurbitacin I (JSI-124) has been shown to overcome resistance to chemotherapy and radiotherapy in vivo [35]. In tumor models with intrinsic or acquired resistance to EGFR tyrosine kinase inhibitors or cetuximab, STAT3 suppression restored the antitumor effects [36]. This suggests that targeting STAT3 may be an efficient therapeutic strategy in NSCLC patients that are not sensitive to EGFR inhibition. Similar results were observed in head and neck squamous cancer cell (HNSCC) tumors recurring after cetuximab treatment, in which increased STAT3 phosphorylation was detected compared with pretreatment biopsies [37]. Moreover, in a pilot study that enrolled patients with NSCLC receiving gefitinib before tumor surgical resection, increased STAT3 phosphorylation was observed in resected tumor tissues [38].

STAT3 blockade may abrogate therapeutic resistance to EGFR and MEK inhibitors. Several STAT3 or JAK inhibitors have been developed, but few have been entered into clinical trials due to either unfavorable chemical properties or lack of biologic activity. Table 1 summarizes some of the STAT3 inhibitors in clinical development, while an extended list of STAT3 inhibitors including repurposing drugs is reviewed by Zhao et al. [26]. OPB-51602 and OPB-31121 are oral compounds, recently investigated in phase I clinical trials, that are thought to inhibit phosphorylation of both STAT3 Tyr705 and Ser727 [39, 40]. AZD9150 is an oligonucleotide antisense molecule (ASO), designed to target the 3 '-untranslated part of STAT3, preventing protein translation [41]. AZD9150 showed antitumor activity in lymphoma and lung cancer models [41]. BBI608 is an orally administered drug that blocks cancer stem cells self-renewal and inhibits STAT3, $\beta$-catenin and Nanog pathways. In a phase I study, BBI608 demonstrated tolerability as well as signs of anti-cancer activity in patients with solid tumors [42]. Finally, eriocalyxin B and evodiamine are STAT3 inhibitors which have not yet reached clinical development stages [43, 44]. Eriocalyxin B, a plant-derived diterpenoid, specifically inhibits phosphorylated Tyr705 but not Ser727. Based on computational modeling analysis, eriocalyxin B selectively interacts with the cysteine 712 located on STAT3 to form a covalent binding and inhibit STAT3 activity [43]. Evodiamine is an alkaloid compound that favors the transcription of SHP-1, a protein tyrosine phosphatase responsible for dephosphorylation of Tyr705, and reduces DNA binding activity and suppression of 
Table 1 Drugs in clinical or preclinical development

\begin{tabular}{|c|c|c|c|c|}
\hline Biomarker & Drug & Molecular mechanism & $\begin{array}{l}\text { Phase of } \\
\text { development }\end{array}$ & References \\
\hline \multirow[t]{6}{*}{ STAT3 } & $\begin{array}{l}\text { OPB-51602 (Otsuka } \\
\text { Pharmaceutical }\end{array}$ & Inhibition of Tyr705 and Ser727 & Phase I completed & {$[39]$} \\
\hline & $\begin{array}{l}\text { OPB-31121 (Otsuka } \\
\text { Pharmaceutical) }\end{array}$ & Inhibition of Tyr705 and Ser727 & Phase I completed & {$[40,107]$} \\
\hline & $\begin{array}{l}\text { AZD9150 } \\
\text { (AstraZeneca) }\end{array}$ & ASO, targeting $3^{\prime}$-untranslated part of STAT3 & Phase I completed & {$[41]$} \\
\hline & $\begin{array}{l}\text { BBI603 (Boston } \\
\text { Biomedical) }\end{array}$ & Inhibition of STAT3, $\beta$-catenin, Nanog & Phase I completed & {$[42]$} \\
\hline & Eriocalyxin B & Inhibition of Tyr705 & Preclinical & {$[43]$} \\
\hline & Evodiamine & $\begin{array}{l}\text { Induction of SHP-1, responsible for Tyr705 } \\
\text { dephosphorylation }\end{array}$ & Preclinical & {$[44]$} \\
\hline \multirow[t]{3}{*}{ PAK } & FRAX-597 & Prevention of ATP binding & Preclinical & {$[58]$} \\
\hline & R-ketorolac & Inhibition of RAC 1 and cdc42 & Repurposing drug & {$[59,60]$} \\
\hline & Ivermectin & PAK1 inhibition in NF2 deficient cells & Preclinical & {$[61]$} \\
\hline \multirow[t]{5}{*}{$\begin{array}{l}\text { Hippo/ } \\
\text { YAP1 }\end{array}$} & mTOR Inhibitors & Double LKB1 and KRAS mutation & $\begin{array}{l}\text { Approved for renal } \\
\text { tumors }\end{array}$ & {$[71]$} \\
\hline & $\begin{array}{l}\text { Selumetinib } \\
\text { (AstraZeneca) }\end{array}$ & NF2 loss and KRAS mutations & Phase II completed & {$[74]$} \\
\hline & $\begin{array}{l}\text { Dasatinib } \\
\text { (Bristol-Myers } \\
\text { Squibb) }\end{array}$ & Inhibition of Src and BCR-ABL & $\begin{array}{l}\text { Approved for } \\
\text { CML and ALL }\end{array}$ & {$[81]$} \\
\hline & Super-TDU & Blockade of YAP-TEAD complex & Preclinical & {$[85]$} \\
\hline & Ivermectin & Blockage of YAP-TEAD complex & Preclinical & {$[86]$} \\
\hline \multirow[t]{5}{*}{ AXL } & $\begin{array}{l}\text { Foretinib } \\
\qquad \text { (GlaxoSimithKline) }\end{array}$ & Inhibition of MET/VEGFR2/AXL & Phase II ongoing & {$[93]$} \\
\hline & Cabozantinib (Exelixis) & Inhibition of MET/VEGFR2/RET/AXL & $\begin{array}{l}\text { Approved for } \\
\text { thyroid cancer }\end{array}$ & {$[93]$} \\
\hline & $\begin{array}{l}\text { MGCD265 (Mirati } \\
\text { Therapeutic) }\end{array}$ & Inhibition of MET/VEGFR2/AXL & Phase I ongoing & {$[93]$} \\
\hline & $\begin{array}{l}\text { BMS-777607 } \\
\text { (Bristol-Myers } \\
\text { Squibb) }\end{array}$ & Inhibition of MET/AXL & Phase I ongoing & {$[93]$} \\
\hline & $\begin{array}{l}\text { BGB324/R428 (BerGen } \\
\text { BIO) }\end{array}$ & Inhibition of AXL & Phase II ongoing & {$[93]$} \\
\hline
\end{tabular}


Table 1 continued

\begin{tabular}{|c|c|c|c|c|}
\hline Biomarker & Drug & Molecular mechanism & $\begin{array}{l}\text { Phase of } \\
\text { development }\end{array}$ & References \\
\hline \multirow[t]{4}{*}{$\mathrm{Hh}$} & GANT61 & Inhibition of Gli1 and Gli2 & Preclinical & {$[97]$} \\
\hline & Genistein & Decreased Gli1 mRNA expression & Preclinical $^{\mathrm{a}}$ & {$[104]$} \\
\hline & EGCG & $\begin{array}{l}\text { Decreased Gli1 mRNA expression and } \\
\text { inhibition of Gli reporter activity }\end{array}$ & Preclinical & {$[104]$} \\
\hline & Mebendazole & Inhibition of Gli1 & Repurposing drug & {$[106]$} \\
\hline
\end{tabular}

${ }^{a}$ Genistein has already been tested in a phase II clinical trial but not as an inhibitor of the Hh pathway

transcription of genes involved in cell cycle progression (cyclin D1), angiogenesis (vascular endothelial growth factor receptor), inflammation (tumor necrosis factor) and programmed cell death (survivin) [44] (Table 1).

\section{PAK PROTEIN KINASES AND THEIR ROLE IN CANCER}

The p21-activated kinase (PAK) family includes six serine/threonine protein kinases, classified into two groups, PAK1-3 (group I) and PAK 4-6 (group II), with PAK1 being the most extensively studied [45]. PAK1 may be deregulated in tumors through gene amplification of the locus 11q13, messenger RNA overexpression or activating mutations [46]. PAK1 can also be hyperactivated by molecular alterations of its upstream regulators. Inactive PAK1 forms an autoinhibited homodimer that is stabilized by interactions between its inhibitory switch domain and the kinase domain. The binding between the small GTPases cell division control protein 42 (Cdc42) or Ras-related C3 botulinum toxin substrate 1 (RAC1) with PAK1 favors the dimer dissociation, resulting in two PAK1 monomers that undergo autophosphorylation and activate downstream signaling [47]. Atypical protein kinase $\mathrm{C}$ isozyme iota
(PKCiota) modulates RAC1 activation [48]. PAK1 regulates several important signaling pathways, including the RAF-MEK-ERK, PI3K-AKT and Wnt- $\beta$-catenin pathways (Fig. 1) [49].

PAK1 is crucial in KRAS mutant tumors. PAK1 activates ERK signaling through CRAF phosphorylation at Ser338 or MEK1 phosphorylation at Ser298 [50]. PAK1 also acts as a scaffold protein that recruits AKT to the plasma membrane allowing its activation (Fig. 1) [51]. In a transgenic mouse model of squamous skin carcinoma harboring KRAS G12D, the lack of PAK1 significantly delayed and reduced tumor development [52]. Biochemical analyses revealed a strong reduction of MEK-ERK and AKT activation in PAK1-negative tumors. However, the available data suggest that ERK has a more determining role in tumorigenesis than AKT, as observed after treatment with ERK and AKT inhibitors. In the same model, RAC1 was found to be a critical player, since the lack of RAC1 is associated with a decreased keratinocyte hyperproliferation and with MEK and AKT activation [53]. PAK1 also phosphorylates CRAF at Ser338. Phosphorylated CRAF translocates into mitochondria and phosphorylates $\mathrm{BAD}$, which prevents the binding to B cell lymphoma-2 (BCL-2), and inhibits apoptosis (Fig. 1) [54]. 
Expression of PAK1 protein has also been analyzed on tissue microarrays of small cell lung cancer (SCLC), lung adenocarcinomas, LSCC and HNSCC. Sixty-four percent of LSCC samples were positive for PAK1 expression, with $52 \%$ of them having moderate or strong staining [55]. Nuclear localization of PAK1 was evident in a significant proportion of LSCC while lung adenocarcinomas and SCLCs expressed weak to moderate levels of PAK1 only in the cytoplasm [55]. Interestingly, epidermal growth factor has been found to stimulate PAK1 activity in HNSCC (Fig. 1) [56]. When PAK1 mRNA expression was examined in a distinct set of 54 laser-capture microdissected lung tissues, PAK1 mRNA expression was highest in LSCC compared with normal lung [55]. PAK1 inhibition in LSCC cell lines determines accumulation in the G1 phase. Levels of the E2F1 transcription factor, which regulates gene expression associated with DNA replication and mitosis and is essential for G1/S progression, were diminished after PAK1 ablation [55]. In LSCC xenograft models, PAK1 inhibition impaired tumor growth, as confirmed by a reduction of Ki-67 expression [55]. PAK1 inhibition alone did not increase apoptosis in NSCLC cells and xenograft models but displayed dramatically enhanced efficacy when combined with apoptosis proteins, EGFR, MEK1/2, or Src inhibitors [55].

Additional studies are warranted to better explore the role of PAK proteins in cancer. Several PAK inhibitors have been synthesized but are still in early stages of clinical development [57]. For instance, PF3758309 was investigated in a phase I study, but development was not continued due to unfavorable pharmacokinetic properties. FRAX-597 is a group I PAK inhibitor, able to prevent ATP binding, with in vivo antitumor activity in a KRAS model [58]. Interestingly, the racemic form of the anti-inflammatory ketorolac, R-ketorolac, beyond cyclooxygenase (COX1/2) inhibition, suppresses RAC1 and Cdc42 [59, 60]. Ivermectin, a well-tolerated antiparasitic macrocyclic lactone has also been found to block PAK1 in human ovarian cancer [61] (Table 1).

\section{THE HIPPO PATHWAY AS A NEW TARGET FOR LUNG CANCER TREATMENT}

The Hippo pathway is a complex signaling system, initially identified in Drosophila and responsible for organ size regulation [62]. In mammals, most components of the Hippo pathway have been implicated as tumor suppressors, including neurofibromin-2 (NF2; also known as merlin), the mammalian sterile 20-like (MST) kinases, the large tumor suppressor homologue (LATS) kinases and the adaptor proteins salvador homolog-1 (SAV1) and Mps one binder kinase activator (MOB). Downstream of LATS are two paralogous transcriptional coactivators: Yes-associated protein-1 (YAP1) and transcriptional coactivator with PDZ-binding motif (TAZ), which drive the expression of numerous effector genes [63]. YAP1 Ser127 phosphorylation by LATS $1 / 2$ draws YAP1/TAZ in the cytoplasm, preventing it from activating TEA domain family member (TEAD)-mediated transcription of connective tissue growth factor (CTGF), AXL or other genes (Fig. 1) [64, 65]. YAP1 can also be activated upon tyrosine phosphorylation by the Src family kinase (SFK) Yes or Jun $\mathrm{N}$ terminal kinases (JUNK) (Fig. 1) [66].

YAP1 is a critical oncogenic KRAS effector and a promising therapeutic target for KRAS mutant tumors [67]. YAP1 induces transcription of the antiapoptotic factor BCL-xL and favors 
resistance to MEK inhibitors. YAP1 suppression in KRAS mutant NSCLC cell lines increases the efficacy of trametinib [67]. In a KRAS mutant pancreatic tumor mice model, YAP1 was found to promote expression of CTGF, cystein-rich angiogenic inducer 61 (CYR61), cyclooxygenase 2 (COX2), matrix metallopeptidase 7 (MMP7), IL-6 and interleukin $1 \alpha$ (IL-1 $\alpha$ ), and induce KRAS mutant pancreatic ductal cell proliferation [68]. ERK was found to be crucial for both phosphorylation and post-transcriptional modifications of YAP1, suggesting that KRAS may directly potentiate the transcriptional activity of YAP1, independent of the Hippo pathway (Fig. 1) [68]. The molecular mechanisms responsible for YAP1 modifications mediated by ERK are not fully characterized, even though multiple ERK phosphorylation motifs have been detected within the YAP1 sequence. To confirm the importance of YAP1 in NSCLC, YAP1 protein expression was measured in a group of NSCLC patients, and high YAP1 levels were observed in patients with KRAS mutant NSCLC [68].

The tumor suppressor liver kinase B1 (LKB1) is an indirect regulator of YAP1 [69]. LKB1 is a serine/threonine kinase, responsible for activation of adenosine monophosphate-activated protein kinase (AMPK) and AMPK related kinases (Fig. 1) [70]. LKB1 knockdown is associated with an increase of YAP1 target genes. LKB1 activates LATS through phosphorylation at threonine 1079 [69]. Indeed, LKB1 controls YAP1 through the regulation of microtubule-associated regulatory kinases (MARKs), a group of proteins implicated in cell polarity and microtubule dynamics that favor the activation of MST1/2 which phosphorylates LATS (Fig. 1) [69]. Furthermore, AMPK activation inhibits the GTP binding protein Ras homolog enriched in brain (RHEB), which suppresses the MAPK signaling and stimulates mTOR (Fig. 1) [71]. Dual inhibition of MEK and mTOR reduced proliferation in NSCLC cells harboring KRAS and LKB1 mutations. LKB1 inactivating mutations are detected in approximately 30\% of patients with adenocarcinoma, and more frequently in patients with KRAS mutant NSCLC [71]. A retrospective analysis showed that the presence of concomitant KRAS and LKB1 mutations is associated with worse patient outcome [72].

NF2 is found mutated in the neurofibromatosis type 2 syndrome [73]. Inactivating mutations of NF2 increase MAPK signaling in KRAS mutant papillary thyroid cancers (PTC), through interactions with PAK1 and YAP1 (Fig. 1). NF2 inhibits RAC1, thus preventing PAK1-mediated phosphorylation of CRAF and MEK [74]. At the same time, NF2 phosphorylates YAP1, thereby blocking its nuclear translocation, and reduces transcription of the RAS genes with a TEAD binding site in their promoter [74]. Lack of NF2, due to loss of chromosome 22q, is detected in $45 \%$ of RAS mutant PTCs and is associated with marked sensitivity to selumetinib [74].

YAP1 overexpression is correlated with lymph node metastasis, pTNM stage and poor prognosis of both lung adenocarcinoma and LSCC [75]. YAP1 has been recognized as a marker of resistance to cetuximab in colorectal cancer patients [76]. YAP1 activation leads to overexpression of EGFR and its ligand amphiregulin (AREG) (Fig. 1) [77, 78]. It has also been demonstrated that extracellular matrix deprivation activates the Hippo pathway and inactivates YAP1 in lung adenocarcinoma, which eventually initiates the squamous trans-differentiation programme [79].

The YAP1-TAZ pathway may be suppressed by compounds that target the upstream Src 
kinase or by small molecules that prevent YAP-TEAD interaction (Table 1). Src inhibitors like saracatinib, dasatinib or bosutinib have been clinically investigated [80]. In vitro data indicate activity not only against Src kinase but also EGFR even though there are few differences in terms of mechanism of action. Saracatinib is the most efficient at inhibiting mutant EGFR, thanks to a mechanism of action independent from Src which is not yet fully understood [81, 82]. Dasatinib exhibits the highest inhibition potential for Src Kinase and wild-type EGFR [83], suggesting that combination with selumetinib or necitumumab could be a very promising treatment strategy for patients with KRAS mutated NSCLC and LSCC, respectively. Small molecules interfering with YAP-TEAD interaction hinder YAP1-induced gene transcription and are also efficient in NF2 inactivation. Verteporfin, currently used in combination with photodynamic therapy to treat macular eye degeneration, was the first investigated YAP1 inhibitor [84]. Super-TDU blocks YAP1-TEAD complexes, mimicking the action of vestigial-like family member 4 (VGLL4), the tumor suppressor that competes with YAP1 for TEAD binding (Fig. 1) [85]. Finally, ivermectin, besides its activity against PAK1 [61], has been identified as a potential YAP1 inhibitor through a mechanism that is not yet clear [86] (Table 1).

\section{AXL: BIOLOGICAL FUNCTIONS AND THERAPEUTIC IMPLICATIONS}

AXL (from the Greek word 'anexelekto,' or uncontrolled), a direct transcriptional target of YAP1, is a tyrosine kinase receptor involved in the epithelial mesenchymal transition (EMT) process [87]. It is a potent oncogene that modulates resistance to conventional and targeted cancer therapies [88]. A gene expression and proteomics-integrated analysis identified an EMT gene signature able to differentiate the epithelial from the mesenchymal phenotype. NSCLC cell lines classified as mesenchymal were resistant to PI3K/AKT inhibitors and exhibited high AXL expression, but great sensitivity to AXL inhibitors [89]. The EMT gene signature classified $60 \%$ of the KRAS mutant cell lines tested in the mesenchymal subgroup. When the signature was applied in patients enrolled in the BATTLE-1 trial (Biomarker integrated Approaches of Targeted Therapies for Lung Cancer Elimination), KRAS mutant patients were equally distributed between the epithelial and the mesenchymal group [89]. These data appear controversial, as preclinical findings suggest that NSCLC cell lines harboring KRAS mutations or cell lines dependent on RAS signal for proliferation are associated with epithelial differentiation, while KRAS mutant cell lines independent of KRAS for survival exhibit mesenchymal behavior [14]. When a KRAS mutant NSCLC patient underwent rebiopsy after 1 week of selumetinib treatment, high expression of the ERBB2/ERBB3 receptors was found, suggesting that heterodimeric complexes involving the ERBB signaling can emerge following MEK inhibition [90]. We were able to identify AXL as the most overexpressed gene in tumors with acquired resistance to erlotinib [88]. In our in vitro and in vivo models, AXL overexpression was related to $\mathrm{NF}-\kappa \mathrm{B}$ activation while knockdown of AXL restored sensitivity to erlotinib treatment in in vivo models [88]. We additionally identified AXL overexpression as a mechanism of acquired resistance to cetuximab in NSCLC and HNSCC [91]. In fact, EGFR directly regulates expression of AXL mRNA through MAPK signaling and the transcription factor c-Jun in cetuximab-resistant cells, creating a positive feedback loop that 
maintains EGFR activation by AXL (Fig. 1) [91]. In addition, AXL blockade inhibits proliferation, migration and invasion in prostate cancer cell lines and in in vivo models through inhibition of the NF- $\kappa \mathrm{B}$ pathway [92]. Intriguingly, AXL blockade inhibits the secretion of IL-6, which in turn reduces proliferation of androgen-insensitive prostate cancer cells via STAT3 inhibition [92].

Several AXL inhibitors are currently under development. Most are ATP competitive kinase inhibitors, with cross-reactivity against multiple tyrosine kinase receptors (Table 1). The lack of AXL kinase domain crystallographic models may explain the difficulties encountered during the development of AXL inhibitors [93]. Foretinib is a dual MET/vascular endothelial growth factor receptor 2 (VEGFR2) inhibitor, with activity against AXL [93]. Cabozantinib, already approved for the treatment of medullary thyroid cancer, inhibits MET, VEGFR2, rearranged during transfection (RET), and AXL [93]. MGCD265 is a MET/VEGFR2 inhibitor also able to suppress AXL activity [93]. BMS-777607 was initially developed as a MET inhibitor but is more potent against AXL. Finally, BGB324/R428, specifically designed to target $\mathrm{AXL}$, is the only compound with two phase I studies ongoing [93].

\section{UNRAVELING THE THERAPEUTIC POTENTIAL OF THE HEDGEHOG PATHWAY IN LUNG CANCER}

The hedgehog (Hh) signaling pathway is highly conserved and has a crucial role in embryogenesis, adult tissue homeostasis and carcinogenesis [94]. Hh signaling is activated following binding of Sonic Hh (Shh), Desert Hh and Indian $\mathrm{Hh}$ ligands to their transmembrane receptor Patched (PTCH), leading to the release of Gli transcription factors by depressing Smoothened (SMO) (Fig. 1) [95].

Ligand-dependent activation of the $\mathrm{Hh}$ pathway has been described in epithelial tumors, but SMO inhibitors have so far failed to show any benefit in clinical trials of pancreatic, colon or ovarian cancer [96, 97]. Complex crosstalk between $\mathrm{Hh}$ and other pathways seems to play a significant role in resistance to $\mathrm{Hh}$ pathway inhibitors. LSCC tumors overexpress PKCiota, SOX2 and $\mathrm{Hh}$ acyltransferase (HHAT) and require PKCiota-SOX2-HHAT signaling to maintain a stem cell phenotype (Fig. 1) [98]. NSCLC cells overexpressing Gli1 can overcome the growth-inhibitory effects of $\mathrm{Hh}$ antagonist such as cyclopamine [99] through crosstalk between the Hh and EGFR signaling pathways. In fact, cooperation between Hedgehog and Gli-EGFR synergistically induces expression of SOX2 [100]. Targeting Hh signaling at the level of Gli may be more effective than targeting either Shh or SMO in LSCC. Gli2 is likely to be the major signaling transducer in LSCC and is the primary activator of Hh signaling, with Gli1 being a transcriptional target of Gli2 [101]. SMO has a minimal role in regulating LSCC survival via the canonical Hh pathway [101]. Loss of SMO did not reduce Gli2 mRNA level in LSCC cell lines and only Gli2 knockdown inhibited cell proliferation and survival and induced apoptosis [101]. The Hh pathway also seems to be involved in KRAS oncogene transformation [102]. Loss of Gli1 inhibits KRAS-induced pancreatic paraneoplastic lesions in mice. Shh promotes binding between Gli1 and the IL- 6 promoter, thus favoring activation of STAT3 and gene transcription (Fig. 1) [102].

Vismodegib is a first-in-class small-molecule SMO inhibitor approved for treatment of advanced basal skin carcinoma [103]. GANT61 is a Gli1 and Gli2 inhibitor [97]. The isoflavone 
genistein also inhibits Gli1 mRNA expression and downregulates Gli reporter activity [104]. Among the catechins contained in green tea, epigallocatechin 3-gallate (EGCG) has been reported to inhibit prostate cancer and chondrosarcoma proliferation through Gli1 inhibition [105]. Finally, mebendazole, a drug approved for treatment of nematode infections, has been found to suppress the formation of the primary cilium, a microtubule-based organelle that functions as a signaling hub for $\mathrm{Hh}$ pathway activation [106].

\section{CONCLUSION: FUTURE PERSPECTIVES}

The success of targeted agents in molecularly defined subsets of patients has radically changed treatment strategies. The emerging issue now is that KRAS mutant and LSCC are not unique entities, as observed in EGFR mutant or EML4-ALK tumors, but heterogeneous diseases in which common signaling pathways dictate distinct pathologic outputs. The characterization of KRAS mutations is not sufficient to properly classify patients. Predictive biomarkers, representative of the underlying activated signaling pathways, need to be defined to develop optimal combinations for synthetic lethal approaches.

\section{ACKNOWLEDGMENTS}

This work was supported by grants from the La Caixa Foundation and Red Tematica de Investigacion Cooperativa en Cancer (RTICC; Grant RD12/0036/0072). All named authors meet the International Committee of Medical Journal Editors (ICMJE) criteria for authorship for this manuscript, take responsibility for the integrity of the work as a whole, and have given final approval for the version to be published.

Disclosures. Dr. Alberto Verlicchi is supported by a Fellowship Award of the European Society for Medical Oncology (ESMO) with the aid of an educational grant from Roche. Dr Sara Pilotto is supported by a Fellowship Award of the International Association for the Study of Lung Cancer (IASLC). Chiara Lazzari, Anastasios Gkountakos, Mariacarmela Santarpia, Imane Chaib, Jose Luis Ramirez Serrano, Santiago Viteri, Daniela Morales Espinosa, Claudio Dazzi, Filippo de Marinis, Peng Cao, Niki Karachaliou, Rafael Rosell have nothing to disclose.

Compliance with Ethics Guidelines This article is based on previously conducted studies and does not involve any new studies of human or animal subjects performed by any of the authors.

Funding Grants from the La Caixa Foundation and Red Tematica de Investigacion Cooperativa en Cancer (RTICC; Grant RD12/0036/0072).

Open Access This article is distributed under the terms of the Creative Commons Attribution-NonCommercial 4.0 International License (http://creativecommons.org/licenses/ by-nc/4.0/), which permits any noncommercial use, distribution, and reproduction in any medium, provided you give appropriate credit to the original author(s) and the source, provide a link to the Creative Commons license, and indicate if changes were made. 


\section{REFERENCES}

1. Lynch TJ, Bell DW, Sordella R, Gurubhagavatula S, Okimoto RA, Brannigan BW, et al. Activating mutations in the epidermal growth factor receptor underlying responsiveness of non-small-cell lung cancer to gefitinib. New Engl J Med. 2004;350(21):2129-39.

2. Mok TS, Wu YL, Thongprasert S, Yang CH, Chu DT, Saijo N, et al. Gefitinib or carboplatin-paclitaxel in pulmonary adenocarcinoma. New Engl J Med. 2009;361(10):947-57.

3. Rosell R, Carcereny E, Gervais R, Vergnenegre A, Massuti B, Felip E, et al. Erlotinib versus standard chemotherapy as first-line treatment for European patients with advanced EGFR mutation-positive non-small-cell lung cancer (EURTAC): a multicentre, open-label, randomised phase 3 trial. Lancet Oncol. 2012;13(3):239-46.

4. Soda M, Choi YL, Enomoto M, Takada S, Yamashita Y, Ishikawa S, et al. Identification of the transforming EML4-ALK fusion gene in non-small-cell lung cancer. Nature. 2007;448 (7153):561-6.

5. Shaw AT, Kim DW, Nakagawa K, Seto T, Crino L, Ahn MJ, et al. Crizotinib versus chemotherapy in advanced ALK-positive lung cancer. New Engl J Med. 2013;368(25):2385-94.

6. $\quad$ Rosell R and Karachaliou N. Large-scale screening for somatic mutations in lung cancer. Lancet. 2016. doi: 10.1016/S0140-6736(15)01125-3.

7. Dearden S, Stevens J, Wu YL, Blowers D. Mutation incidence and coincidence in non small-cell lung cancer: meta-analyses by ethnicity and histology (mutMap). Ann Oncol. 2013;24(9):2371-6.

8. Nussinov R, Tsai CJ, Chakrabarti M, Jang H. A new view of ras isoforms in cancers. Cancer Res. 2016;76(1):18-23.

9. Mascaux C, Iannino N, Martin B, Paesmans M, Berghmans T, Dusart $M$, et al. The role of RAS oncogene in survival of patients with lung cancer: a systematic review of the literature with meta-analysis. Br J Cancer. 2005;92(1):131-9.

10. Yu HA, Sima CS, Shen R, Kass S, Gainor J, Shaw A, et al. Prognostic impact of KRAS mutation subtypes in 677 patients with metastatic lung adenocarcinomas. J Thor Oncol. 2015;10(3):431-7.

11. Pylayeva-Gupta Y, Grabocka E, Bar-Sagi D. RAS oncogenes: weaving a tumorigenic web. Nat Rev Cancer. 2011;11(11):761-74.
12. Janne PA, Shaw AT, Pereira JR, Jeannin G, Vansteenkiste J, Barrios C, et al. Selumetinib plus docetaxel for KRAS-mutant advanced non-small-cell lung cancer: a randomised, multicentre, placebo-controlled, phase 2 study. Lancet Oncol. 2013;14(1):38-47.

13. Ihle NT, Byers LA, Kim ES, Saintigny P, Lee JJ, Blumenschein GR, et al. Effect of KRAS oncogene substitutions on protein behavior: implications for signaling and clinical outcome. J Natl Cancer Inst. 2012;104(3):228-39.

14. Singh A, Greninger P, Rhodes D, Koopman L, Violette S, Bardeesy $\mathrm{N}$, et al. A gene expression signature associated with "K-Ras addiction" reveals regulators of EMT and tumor cell survival. Cancer Cell. 2009;15(6):489-500.

15. Skoulidis F, Byers LA, Diao L, Papadimitrakopoulou VA, Tong P, Izzo J, et al. Co-occurring genomic alterations define major subsets of KRAS-mutant lung adenocarcinoma with distinct biology, immune profiles, and therapeutic vulnerabilities. Cancer Discov. 2015;5(8):860-77.

16. Cheng H, Shcherba M, Kandavelou K, Liang Y, Liu $\mathrm{H}$, Perez-Soler R. Emerging drugs for squamous cell lung cancer. Expert Opin Emerg drugs. 2015;20(1):149-60.

17. Besse B, Adjei A, Baas P, Meldgaard P, Nicolson M, Paz-Ares L, et al. 2nd ESMO Consensus Conference on Lung Cancer: non-small-cell lung cancer first-line/second and further lines of treatment in advanced disease. Ann Oncol. 2014;25(8):1475-84.

18. Brahmer J, Reckamp KL, Baas P, Crino L, Eberhardt WE, Poddubskaya E, et al. Nivolumab versus docetaxel in advanced squamous-cell non-small-cell lung cancer. New Engl J Med. 2015;373(2):123-35.

19. Gandara DR, Hammerman PS, Sos ML, Lara PN Jr, Hirsch FR. Squamous cell lung cancer: from tumor genomics to cancer therapeutics. Clin Cancer Res. 2015;21(10):2236-43.

20. Lopez-Malpartida AV, Ludena MD, Varela G, Pichel JG. Differential ErbB receptor expression and intracellular signaling activity in lung adenocarcinomas and squamous cell carcinomas. Lung Cancer. 2009;65(1):25-33.

21. Pirker R, Pereira JR, Szczesna A, von Pawel J, Krzakowski M, Ramlau R, et al. Cetuximab plus chemotherapy in patients with advanced non-small-cell lung cancer (FLEX): an open-label randomised phase III trial. Lancet. 2009;373 (9674):1525-31. 
22. Pirker R, Pereira JR, von Pawel J, Krzakowski M, Ramlau R, Park K, et al. EGFR expression as a predictor of survival for first-line chemotherapy plus cetuximab in patients with advanced non-small-cell lung cancer: analysis of data from the phase 3 FLEX study. Lancet Oncol. 2012;13(1):33-42.

23. Lynch TJ, Patel T, Dreisbach L, McCleod M, Heim WJ, Hermann RC, et al. Cetuximab and first-line taxane/carboplatin chemotherapy in advanced non-small-cell lung cancer: results of the randomized multicenter phase III trial BMS099. J Clin Oncol. 2010;28(6):911-7.

24. Thatcher N, Hirsch FR, Luft AV, Szczesna A, Ciuleanu TE, Dediu M, et al. Necitumumab plus gemcitabine and cisplatin versus gemcitabine and cisplatin alone as first-line therapy in patients with stage IV squamous non-small-cell-lung cancer (SQUIRE): an open-label, randomized, controlled phase 3 trial. Lancet Oncol. 2015;16(7):763-74.

25. Perez-Moreno P, Brambilla E, Thomas R, Soria JC. Squamous cell carcinoma of the lung: molecular subtypes and therapeutic opportunities. Clin Cancer Res. 2012;18(9):2443-51.

26. Zhao C, Li H, Lin HJ, Yang S, Lin J, Liang G. Feedback activation of STAT3 as a cancer drug-resistance mechanism. Trends Pharmacol Sci. 2016;37(1):47-61.

27. Ihle JN. STATs: signal transducers and activators of transcription. Cell. 1996;84(3):331-4.

28. Lee HJ, Zhuang G, Cao Y, Du P, Kim HJ, Settleman J. Drug resistance via feedback activation of Stat3 in oncogene-addicted cancer cells. Cancer Cell. 2014;26(2):207-21.

29. Brooks GD, McLeod L, Alhayyani S, Miller A, Russell PA, Ferlin W, et al. IL-6 trans-signaling promotes KRAS-driven lung carcinogenesis. Cancer Res. 2016;76(4):866-76.

30. Gough DJ, Corlett A, Schlessinger K, Wegrzyn J, Larner AC, Levy DE. Mitochondrial STAT3 supports Ras-dependent oncogenic transformation. Science. 2009;324(5935):1713-6.

31. Gough DJ, Koetz L, Levy DE. The MEK-ERK pathway is necessary for serine phosphorylation of mitochondrial STAT3 and Ras-mediated transformation. PLoS ONE. 2013;8(11):e83395.

32. Wegrzyn J, Potla R, Chwae YJ, Sepuri NB, Zhang Q, Koeck T, et al. Function of mitochondrial Stat3 in cellular respiration. Science. 2009;323(5915):793-7.

33. Tammineni $\mathrm{P}$, Anugula $\mathrm{C}$, Mohammed F, Anjaneyulu M, Larner AC, Sepuri NB. The import of the transcription factor STAT3 into mitochondria depends on GRIM-19, a component of the electron transport chain. J Biol Chem. 2013;288(7):4723-32.

34. Kim HS, Park YH, Lee J, Ahn JS, Kim J, Shim YM, et al. Clinical impact of phosphorylated signal transducer and activator of transcription 3, epidermal growth factor receptor, p53, and vascular endothelial growth factor receptor 1 expression in resected adenocarcinoma of lung by using tissue microarray. Cancer. 2010;116(3): 676-85.

35. Hsu HS, Huang PI, Chang YL, Tzao C, Chen YW, Shih HC, et al. Cucurbitacin I inhibits tumorigenic ability and enhances radiochemosensitivity in nonsmall cell lung cancer-derived CD133-positive cells. Cancer. 2011;117(13): 2970-85.

36. Chiu HC, Chou DL, Huang CT, Lin WH, Lien TW, Yen KJ, et al. Suppression of Stat3 activity sensitizes gefitinib-resistant non small cell lung cancer cells. Biochem Pharmacol. 2011;81(11): 1263-70.

37. Sen M, Joyce S, Panahandeh M, Li C, Thomas SM, Maxwell J, et al. Targeting Stat3 abrogates EGFR inhibitor resistance in cancer. Clin Cancer Res. 2012;18(18):4986-96.

38. Haura EB, Sommers E, Song L, Chiappori A, Becker A. A pilot study of preoperative gefitinib for early-stage lung cancer to assess intratumor drug concentration and pathways mediating primary resistance. J Thor Oncol. 2010;5(11):1806-14.

39. Wong AL, Soo RA, Tan DS, Lee SC, Lim JS, Marban PC, et al. Phase I and biomarker study of OPB-51602, a novel signal transducer and activator of transcription (STAT) 3 inhibitor, in patients with refractory solid malignancies. Ann Oncol. 2015;26(5):998-1005.

40. Bendell JC, Hong DS, Burris HA 3rd, Naing A, Jones SF, Falchook G, et al. Phase 1, open-label, dose-escalation, and pharmacokinetic study of STAT3 inhibitor OPB-31121 in subjects with advanced solid tumors. Cancer Chemother Pharmacol. 2014;74(1):125-30.

41. Hong D, Kurzrock R, Kim Y, Woessner R, Younes A, Nemunaitis J, et al. AZD9150, a next-generation antisense oligonucleotide inhibitor of STAT3 with early evidence of clinical activity in lymphoma and lung cancer. Sci Transl Med. 2015;7(314): 314 ra185.

42. Jonker DJ, Stephenson J, Edenfield WJ, Supko JG, Li Y, Li W, Hitron M, Leggett D, Kerstein D, Li C. A phase I extension study of BBI608, a first-in-class 
cancer stem cell (CSC) inhibitor, in patients with advanced solid tumors. J Clin Oncol. 2014;32:5s (suppl; abstr 2546).

43. Yu X, He L, Cao P, Yu Q. Eriocalyxin B inhibits STAT3 signaling by covalently targeting STAT3 and blocking phosphorylation and activation of STAT3. PLoS ONE. 2015;10(5):e0128406.

44. Yang J, Cai X, Lu W, Hu C, Xu X, Yu Q, et al. Evodiamine inhibits STAT3 signaling by inducing phosphatase shatterproof 1 in hepatocellular carcinoma cells. Cancer Lett. 2013;328(2):243-51.

45. Arias-Romero LE, Chernoff J. A tale of two Paks. Biol Cell. 2008;100(2):97-108.

46. Radu M, Semenova G, Kosoff R, Chernoff J. PAK signalling during the development and progression of cancer. Nat Rev Cancer. 2014;14(1):13-25.

47. Parrini MC, Lei M, Harrison SC, Mayer BJ. Pak1 kinase homodimers are autoinhibited in trans and dissociated upon activation by Cdc42 and Rac1. Mol Cell. 2002;9(1):73-83.

48. Regala RP, Weems C, Jamieson L, Khoor A, Edell ES, Lohse CM, et al. Atypical protein kinase C iota is an oncogene in human non-small cell lung cancer. Cancer Res. 2005;65(19):8905-11.

49. Arias-Romero LE, Villamar-Cruz O, Huang M, Hoeflich KP, Chernoff J. Pak1 kinase links ErbB2 to beta-catenin in transformation of breast epithelial cells. Cancer Res. 2013;73(12):3671-82.

50. Balasenthil S, Sahin AA, Barnes CJ, Wang RA, Pestell RG, Vadlamudi RK, et al. p21-activated kinase-1 signaling mediates cyclin D1 expression in mammary epithelial and cancer cells. J Biol Chem. 2004;279(2):1422-8.

51. Higuchi M, Onishi K, Kikuchi C, Gotoh Y. Scaffolding function of PAK in the PDK1-Akt pathway. Nat Cell Biol. 2008;10(11):1356-64.

52. Chow HY, Jubb AM, Koch JN, Jaffer ZM, Stepanova $\mathrm{D}$, Campbell DA, et al. p21-Activated kinase 1 is required for efficient tumor formation and progression in a Ras-mediated skin cancer model. Cancer Res. 2012;72(22):5966-75.

53. Wang Z, Pedersen E, Basse A, Lefever T, Peyrollier $\mathrm{K}$, Kapoor S, et al. Rac1 is crucial for Ras-dependent skin tumor formation by controlling Pak1-Mek-Erk hyperactivation and hyperproliferation in vivo. Oncogene. 2010;29(23):3362-73.

54. Wu X, Carr HS, Dan I, Ruvolo PP, Frost JA. p21 activated kinase 5 activates Raf- 1 and targets it to mitochondria. J Cell Biochem. 2008;105(1):167-75.
55. Ong CC, Jubb AM, Haverty PM, Zhou W, Tran V, Truong $\mathrm{T}$, et al. Targeting p21-activated kinase 1 (PAK1) to induce apoptosis of tumor cells. Proc Natl Acad Sci USA. 2011;108(17):7177-82.

56. Yang Z, Bagheri-Yarmand R, Wang RA, Adam L, Papadimitrakopoulou VV, Clayman GL, et al. The epidermal growth factor receptor tyrosine kinase inhibitor ZD1839 (Iressa) suppresses c-Src and Pak1 pathways and invasiveness of human cancer cells. Clin Cancer Res. 2004;10(2):658-67.

57. Rudolph J, Crawford JJ, Hoeflich KP, Wang W. Inhibitors of p21-activated kinases (PAKs). J Med Chem. 2015;58(1):111-29.

58. Yeo D, He H, Baldwin G, Nikfarjam M. FRAX597, a PAK1 inhibitor, synergises with gemcitabine in the reduction of pancreatic cancer growth. Ann Oncol. 2015;26(suppl 4):v10-iv11.

59. Guo Y, Kenney SR, Muller CY, Adams S, Rutledge $\mathrm{T}$, Romero E, et al. R-ketorolac targets Cdc42 and Rac1 and alters ovarian cancer cell behaviors critical for invasion and metastasis. Mol Cancer Ther. 2015;14(10):2215-27.

60. Guo Y, Kenney SR, Cook L, Adams SF, Rutledge T, Romero E, et al. A novel pharmacologic activity of ketorolac for therapeutic benefit in ovarian cancer patients. Clin Cancer Res. 2015;21(22):5064-72.

61. Hashimoto H, Messerli SM, Sudo T, Maruta H. Ivermectin inactivates the kinase PAK1 and blocks the PAK1-dependent growth of human ovarian cancer and NF2 tumor cell lines. Drug Discov Ther. 2009;3(6):243-6.

62. Yu FX, Zhao B, Guan KL. Hippo pathway in organ size control, tissue homeostasis, and cancer. Cell. 2015;163(4):811-28.

63. Zhang H, Pasolli HA, Fuchs E. Yes-associated protein (YAP) transcriptional coactivator functions in balancing growth and differentiation in skin. Proc Natl Acad Sci USA. 2011;108(6):2270-5.

64. Zhang L, Ren F, Zhang Q, Chen Y, Wang B, Jiang J. The TEAD/TEF family of transcription factor Scalloped mediates Hippo signaling in organ size control. Dev Cell. 2008;14(3):377-87.

65. Taniguchi $\mathrm{K}, \mathrm{Wu} \mathrm{LW}$, Grivennikov SI, de Jong PR, Lian I, Yu FX, et al. A gp130-Src-YAP module links inflammation to epithelial regeneration. Nature. 2015;519(7541):57-62.

66. Varelas X. The Hippo pathway effectors TAZ and YAP in development, homeostasis and disease. Development. 2014;141(8):1614-26. 
67. Lin L, Sabnis AJ, Chan E, Olivas V, Cade L, Pazarentzos E, et al. The Hippo effector YAP promotes resistance to RAF- and MEK-targeted cancer therapies. Nat Genet. 2015;47(3):250-6.

68. Zhang W, Nandakumar N, Shi Y, Manzano M, Smith A, Graham G, et al. Downstream of mutant KRAS, the transcription regulator YAP is essential for neoplastic progression to pancreatic ductal adenocarcinoma. Sci Signal. 2014;7(324):ra42.

69. Mohseni M, Sun J, Lau A, Curtis S, Goldsmith J, Fox VL, et al. A genetic screen identifies an LKB1-MARK signalling axis controlling the Hippo-YAP pathway. Nat Cell Biol. 2014;16(1):108-17.

70. Katajisto P, Vallenius T, Vaahtomeri K, Ekman N, Udd L, Tiainen $M$, et al. The LKB1 tumor suppressor kinase in human disease. Biochim Biophys Acta. 2007;1775(1):63-75.

71. Mahoney CL, Choudhury B, Davies H, Edkins S, Greenman C, Haaften G, et al. LKB1/KRAS mutant lung cancers constitute a genetic subset of NSCLC with increased sensitivity to MAPK and mTOR signalling inhibition. $\mathrm{Br} \mathrm{J}$ Cancer. 2009;100(2):370-5.

72. Calles A, Sholl LM, Rodig SJ, Pelton AK, Hornick $\mathrm{JL}$, Butaney M, et al. Immunohistochemical loss of LKB1 Is a biomarker for more aggressive biology in KRAS-mutant lung adenocarcinoma. Clin Cancer Res. 2015;21(12):2851-60.

73. Rouleau GA, Merel P, Lutchman M, Sanson M, Zucman J, Marineau C, et al. Alteration in a new gene encoding a putative membrane-organizing protein causes neuro-fibromatosis type 2 . Nature. 1993;363(6429):515-21.

74. Garcia-Rendueles ME, Ricarte-Filho JC, Untch BR, Landa I, Knauf JA, Voza F, et al. NF2 loss promotes oncogenic RAS-induced thyroid cancers via YAP-dependent transactivation of RAS proteins and sensitizes them to MEK inhibition. Cancer Discov. 2015;5(11):1178-93.

75. Wang Y, Dong Q, Zhang Q, Li Z, Wang E, Qiu X. Overexpression of yes-associated protein contributes to progression and poor prognosis of non-small-cell lung cancer. Cancer Sci. 2010;101(5):1279-85.

76. Lee KW, Lee SS, Kim SB, Sohn BH, Lee HS, Jang HJ, et al. Significant association of oncogene YAP1 with poor prognosis and cetuximab resistance in colorectal cancer patients. Clin Cancer Res. 2015;21(2):357-64.

77. Song S, Honjo S, Jin J, Chang SS, Scott AW, Chen $\mathrm{Q}$, et al. The hippo coactivator YAP1 mediates
EGFR overexpression and confers chemoresistance in esophageal cancer. Clin Cancer Res. 2015;21(11):2580-90.

78. Liu AM, Xu MZ, Chen J, Poon RT, Luk JM. Targeting YAP and Hippo signaling pathway in liver cancer. Expert Opin Ther Targets. 2010;14(8):855-68.

79. Gao Y, Zhang W, Han X, Li F, Wang X, Wang R, et al. YAP inhibits squamous transdifferentiation of Lkb1-deficient lung adenocarcinoma through ZEB2-dependent DNp63 repression. Nature Commun. 2014;5:4629.

80. Roskoski R Jr. Src protein-tyrosine kinase structure, mechanism, and small molecule inhibitors. Pharmacol Res. 2015;94:9-25.

81. Formisano L, D'Amato V, Servetto A, Brillante S, Raimondo L, Di Mauro C, et al. Src inhibitors act through different mechanisms in non-small cell lung cancer models depending on EGFR and RAS mutational status. Oncotarget. 2015;6(28):26090-103.

82. Green TP, Fennell M, Whittaker R, Curwen J, Jacobs V, Allen J, et al. Preclinical anticancer activity of the potent, oral Src inhibitor AZD0530. Mol Oncol. 2009;3(3):248-61.

83. Nautiyal J, Majumder P, Patel BB, Lee FY, Majumdar AP. Src inhibitor dasatinib inhibits growth of breast cancer cells by modulating EGFR signaling. Cancer Lett. 2009;283(2):143-51.

84. Liu-Chittenden Y, Huang B, Shim JS, Chen Q, Lee SJ, Anders RA, et al. Genetic and pharmacological disruption of the TEAD-YAP complex suppresses the oncogenic activity of YAP. Genes Dev. 2012;26(12):1300-5.

85. Jiao S, Wang H, Shi Z, Dong A, Zhang W, Song X, et al. A peptide mimicking VGLL4 function acts as a YAP antagonist therapy against gastric cancer. Cancer Cell. 2014;25(2):166-80.

86. Nishio $M$, Sugimachi $K$, Goto $H$, Wang J, Morikawa T, Miyachi Y, et al. Dysregulated YAP1/TAZ and TGF-beta signaling mediate hepatocarcinogenesis in Mob1a/1b-deficient mice. Proc Natl Acad Sci USA. 2016;113(1):E71-80.

87. Xu MZ, Chan SW, Liu AM, Wong KF, Fan ST, Chen $\mathrm{J}$, et al. AXL receptor kinase is a mediator of YAP-dependent oncogenic functions in hepatocellular carcinoma. Oncogene. 2011;30(10): 1229-40.

88. Zhang $\mathrm{Z}$, Lee JC, Lin L, Olivas $\mathrm{V}$, Au V, LaFramboise $\mathrm{T}$, et al. Activation of the AXL kinase causes resistance to EGFR-targeted therapy in lung cancer. Nat Genet. 2012;44(8):852-60. 
89. Byers LA, Diao L, Wang J, Saintigny P, Girard L, Peyton M, et al. An epithelial-mesenchymal transition gene signature predicts resistance to EGFR and PI3K inhibitors and identifies Axl as a therapeutic target for overcoming EGFR inhibitor resistance. Clin Cancer Res. 2013;19(1):279-90.

90. Sun C, Hobor S, Bertotti A, Zecchin D, Huang S, Galimi $\mathrm{F}$, et al. Intrinsic resistance to MEK inhibition in KRAS mutant lung and colon cancer through transcriptional induction of ERBB3. Cell Rep. 2014;7(1):86-93.

91. Elkabets $M$, Pazarentzos E, Juric D, Sheng $Q$, Pelossof RA, Brook S, et al. AXL mediates resistance to PI3Kalpha inhibition by activating the EGFR/PKC/mTOR axis in head and neck and esophageal squamous cell carcinomas. Cancer Cell. $2015 ; 27(4): 533-46$.

92. Paccez JD, Vasques GJ, Correa RG, Vasconcellos JF, Duncan K, Gu X, et al. The receptor tyrosine kinase Axl is an essential regulator of prostate cancer proliferation and tumor growth and represents a new therapeutic target. Oncogene. 2013;32(6):689-98.

93. Myers SH, Brunton VG, Unciti-Broceta A. AXL inhibitors in cancer: a medicinal chemistry perspective. J Med Chem. 2015. doi:10.1021/acs. jmedchem.5b01273.

94. Lum L, Beachy PA. The Hedgehog response network: sensors, switches, and routers. Science. 2004;304(5678):1755-9.

95. Basset-Seguin N, Sharpe HJ, de Sauvage FJ. Efficacy of Hedgehog pathway inhibitors in Basal cell carcinoma. Mol Cancer Ther. 2015;14(3):633-41.

96. Tian H, Callahan CA, DuPree KJ, Darbonne WC, Ahn CP, Scales SJ, et al. Hedgehog signaling is restricted to the stromal compartment during pancreatic carcinogenesis. Proc Natl Acad Sci USA. 2009;106(11):4254-9.

97. Gonnissen A, Isebaert S, Haustermans K. Targeting the Hedgehog signaling pathway in cancer: beyond Smoothened. Oncotarget. 2015;6(16):13899-913.

98. Justilien V, Walsh MP, Ali SA, Thompson EA, Murray NR, Fields AP. The PRKCI and SOX2 oncogenes are coamplified and cooperate to activate Hedgehog signaling in lung squamous cell carcinoma. Cancer Cell. 2014;25(2):139-51.

99. Yuan Z, Goetz JA, Singh S, Ogden SK, Petty WJ, Black CC, et al. Frequent requirement of hedgehog signaling in non-small cell lung carcinoma. Oncogene. 2007;26(7):1046-55.

100. Rosell R, Bivona TG, Karachaliou N. Genetics and biomarkers in personalisation of lung cancer treatment. Lancet. 2013;382(9893):720-31.

101. Huang L, Walter V, Hayes DN, Onaitis $M$. Hedgehog-GLI signaling inhibition suppresses tumor growth in squamous lung cancer. Clin Cancer Res. 2014;20(6):1566-75.

102. Mills LD, Zhang Y, Marler RJ, Herreros-Villanueva M, Zhang L, Almada LL, et al. Loss of the transcription factor GLI1 identifies a signaling network in the tumor microenvironment mediating KRAS oncogene-induced transformation. J Biol Chem. 2013;288(17):11786-94.

103. Sekulic A, Migden MR, Oro AE, Dirix L, Lewis KD, Hainsworth JD, et al. Efficacy and safety of vismodegib in advanced basal-cell carcinoma. New Engl J Med. 2012;366(23):2171-9.

104. Slusarz A, Shenouda NS, Sakla MS, Drenkhahn SK, Narula AS, MacDonald RS, et al. Common botanical compounds inhibit the hedgehog signaling pathway in prostate cancer. Cancer Res. 2010;70(8):3382-90.

105. Tang GQ, Yan TQ, Guo W, Ren TT, Peng CL, Zhao $\mathrm{H}$, et al. (-)-Epigallocatechin-3-gallate induces apoptosis and suppresses proliferation by inhibiting the human Indian Hedgehog pathway in human chondrosarcoma cells. J Cancer Res Clin Oncol. 2010;136(8):1179-85.

106. Larsen AR, Bai RY, Chung JH, Borodovsky A, Rudin CM, Riggins GJ, et al. Repurposing the antihelmintic mebendazole as a hedgehog inhibitor. Mol Cancer Ther. 2015;14(1):3-13.

107. Oh DY, Lee SH, Han SW, Kim MJ, Kim TM, Kim TY, et al. Phase I Study of OPB-31121, an Oral STAT3 Inhibitor, in Patients with Advanced Solid Tumors. Cancer Res Treat. 2015;47(4):607-15. 